\title{
First Steps Towards Full Model Based Motion Planning and Control of Quadrupeds: A Hybrid Zero Dynamics Approach
}

\author{
Wen-Loong $\mathrm{Ma}^{1}$, Kaveh Akbari Hamed ${ }^{2}$ and Aaron D. Ames ${ }^{3}$
}

\begin{abstract}
The hybrid zero dynamics (HZD) approach has become a powerful tool for the gait planning and control of bipedal robots. This paper aims to extend the HZD methods to address walking, ambling and trotting behaviors on a quadrupedal robot. We present a framework that systematically generates a wide range of optimal trajectories and then provably stabilizes them for the full-order, nonlinear and hybrid dynamical models of quadrupedal locomotion. The gait planning is addressed through a scalable nonlinear programming using direct collocation and HZD. The controller synthesis for the exponential stability is then achieved through the Poincaré sections analysis. In particular, we employ an iterative optimization algorithm involving linear and bilinear matrix inequalities (LMIs and BMIs) to design HZD-based controllers that guarantee the exponential stability of the fixed points for the Poincaré return map. The power of the framework is demonstrated through gait generation and HZDbased controller synthesis for an advanced quadruped robot, -Vision 60, with 36 state variables and 12 control inputs. The numerical simulations as well as real world experiments confirm the validity of the proposed framework.
\end{abstract}

\section{INTRODUCTION}

Quadrupedal locomotion has a long and rich history of outstanding agility and dynamic stability without formal analysis, thanks to the multi-support nature of such systems. Some famous quadrupedal examples include, but are not limited to, the BigDog [28], Minitaur [11], ANYmal [18] and Cheetah robot [9]. State-of-the-art approaches for the controls and planning of quadrupeds mainly utilize model reduction to partly mitigate the computational complexity of the full-order techniques arising from nonlinearity and hybrid nature of models. For instance, massless legs, the linear inverted pendulum model [21] and planar motion planning are often utilized assumptions. While they do have many implementation advantages, one needs to design controllers that overcome the uncertainty induced by the difference between modeling and reality. Further, formal guarantees on stability are rarely studied in quadrupedal research.

On the other hand, the hybrid system approaches have become a powerful tool for modeling the dynamics of bipedal locomotion, in which, steady state locomotion corresponds

*The work of WL. Ma and A. D. Ames are supported by NSF grants 1724464, 1544332 and 1724457. The work of K. Akbari Hamed is supported by the NSF grants 1854898 and 1906727 .

${ }^{1} \mathrm{WL}$. Ma is with the department of mechanical engineering, California Institute of Technology, Pasadena, CA, 91125. wma@caltech. edu

${ }^{2} \mathrm{~K}$. Akbari Hamed is with the faulty of mechanical engineering, Virginia Polytechnic Institute and State University, Blacksburg, VA 24061 kavehakbarihamed@vt.edu

${ }^{3} \mathrm{~A}$. Ames is with the faculty of the department of Control + Dynamical Systems, California Institute of Technology, Pasadena, CA, 91125. amesccaltech.edu

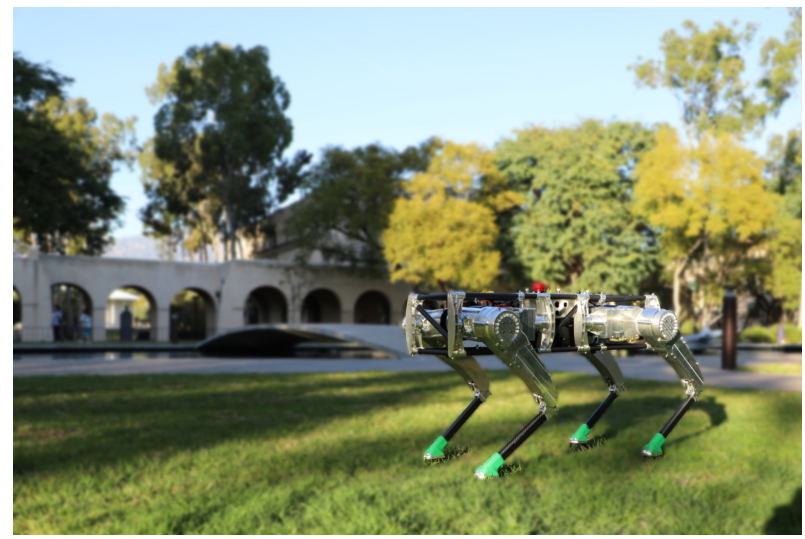

Fig. 1. The Vision 60-v3.2 robot from Ghost Robotics.

to periodic solutions of these hybrid dynamical systems. One of the hybrid control approaches is the hybrid zero dynamics (HZD) framework [31]. HZD is an extension of the notion of Byrnes-Isidori zero dynamics [19] to hybrid models of locomotion for which the resultant zero dynamics manifolds are invariant under the continuous- and discretetime dynamics. HZD has been successful for designing gaits for bipedal locomotion and provide experiment-level controllers, see e.g., [7], [30], [2], [10], [27], [20], but has not yet been applied to the control of quadrupeds. The challenge in computation and controls mainly rises from the increased degrees of freedom (DOF) and richer contact scenarios of quadrupeds over bipeds.

The main objective of this paper is to introduce an alternative approach to contemporary control schemes of quadrupeds that are mainly based on simplified models, and to make the first steps towards extending the results of HZD framework from stability critical systems such as bipeds to a more complicated but more robust system - the quadrupedal robot. We model their dynamics as a hybrid system, optimize trajectories via a HZD optimization algorithm, analyze the dynamic stability via the Poincaré return map and then synthesize HZD-based controllers with iterative optimization problems including linear and bilinear matrix inequalities (LMIs and BMIs). The theoretically stable controller is validated with experiments on a quadrupedal behavior, ambling on the Vision 60 robot (Fig. 11). This result suggests a new approach to realize dynamically stable behaviors such as ambling, trotting and galloping in experiments.

This paper is organized as follows: Section II presents a hybrid dynamic model, based on which we design a nonlinear controller and trajectory optimization algorithm, 


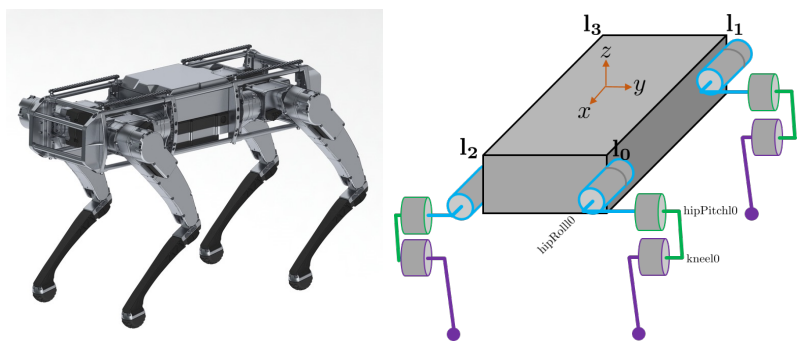

Fig. 2. On the left is the rendering of the robot design, and on the right is the illustration of the configuration coordinates for the robot. The leg indices $l_{*}$ are shown on the vertices of the body link. Each leg has three actuated joints and equipped with a point foot.

known as the HZD optimization. Using this framework, we generate gaits including walking, ambling and trotting in simulation. Section III addresses the exponential stabilization problem as well as the HZD-based controller synthesis via BMIs. Section IV validates the ambling controllers with experiments. A comprehensive comparison is provided for the results. Section V contains conclusions and future directions. This paper presents a self-contained approach that works in both simulation and experiment, and serves as the first steps towards designing full model based controllers for quadrupedal dynamic locomotion.

\section{HYBRID MOTION PLANNING}

We consider the nonlinear model of quadrupedal locomotion as a hybrid dynamical system, which is an alternating sequence of continuous- and discrete-time dynamics. The order of the sequence is dictated by contact events. In comparison with bipedal walking, the increased number of contact points of quadrupeds increased the complexity of the hybrid model substantially. In this section, we introduce a unified model for quadrupedal behaviors including walking, ambling and trotting, based on which we design full model based optimal controllers as well as simulation validation.

\section{A. The robot}

The robot we study in this paper is the Vision 60-v3.2 (see Fig. 1), a quadrupedal robot built by Ghost Robotics, with the total weight of $26 \mathrm{~kg}$ and maximum standing height of $0.5 \mathrm{~m}$. As shown in Fig. 2, we model the quadruped as a 13-link system: a body link and four legs, each of which has three children links - the hip, upper and lower links. The configuration variables of Vision 60 are denoted by $q \in$ $\mathcal{Q} \subset \mathbb{R}^{n}$ where $n=18$ is the total number of degrees of freedom (DOF) without considering any contact constraints. Utilizing the floating base convention [15], we can have $q^{T}=$ $\left(q_{b}^{T}, q_{l}^{T}\right)$, in which $q_{b} \in \mathbb{R}^{3} \times \mathrm{SO}(3)$ represents the global Cartesian position and orientation of a frame attached to the body linkage, and the local coordinates $q_{l} \in \mathbb{R}^{12}$ denote the 12 joint variables: hip roll, hip pitch and knee angles. These angles are denoted by $\theta_{h r_{j}}, \theta_{h p_{j}}, \theta_{k_{j}}$ for the $j$-th leg, all of which are actuated by Brushless (BL) DC motors. This yields the system's total DOF to be 18 and control inputs $u \in \mathbb{R}^{12}$. With different scenarios of foot contacts with the ground, we have a mixture of overactuated, fully-actuated, and underactuated domains (i.e., phases) for the dynamics. Futher, we can define the state space $\mathcal{X}=T \mathcal{Q} \subseteq \mathbb{R}^{2 n}$ with the state vector $x^{T}=\left(q^{T}, \dot{q}^{T}\right)$, where $T \mathcal{Q}$ is the tangent bundle of the configuration space $\mathcal{Q}$.

We now define the different modes of the quadrupedal dynamics, separated by discrete events. This fusion of continuous- and discrete-time dynamics yields a hybrid automaton model [12]. For more detailed definitions of hybrid modeling for bipedal robots, we refer the readers to [8].

\section{B. The continuous-time domain: constrained dynamics}

Given the floating base coordinates, we can derive the unconstrained dynamics, i.e. without any contact constraints, by the Euler-Lagrange equations as:

$$
D(q) \ddot{q}+H(q, \dot{q})=B u
$$

where $D(q) \in \mathbb{R}^{n \times n}$ is the mass-inertia matrix, $H(q, \dot{q}) \in$ $\mathbb{R}^{n}$ includes the Coriolis, centrifugal and gravity terms and $B(q) \in \mathbb{R}^{n \times m}$ is the actuation matrix and $u \in \mathbb{R}^{m}$ is the torque applied at joints with $m=12$.

Now consider $k \in\{1,2,3,4\}$ feet standing on the ground, which means $\bar{k}=4-k$ feet are swinging in the air. This creates a variety of contact scenarios, hence generates different types of quadrupedal behaviors (see Fig. 3). For each contact situation, we associate a continuous domain:

$$
\mathcal{D}_{v}:=\left\{(x, u) \mid h_{v}(q)=\dot{h}_{v}(q, \dot{q})=0, N_{v} \succeq 0, h_{\mathrm{s}, v}(q) \succeq 0\right\}
$$

where $v \in\{1,2,3 \ldots\}$ is the domain index and $h_{v}(q) \in \mathbb{R}^{k}$ is the height of all standing feet with ground reaction force $N_{v}(x, u) \in \mathbb{R}^{k}$. The height of the other feet, referred as the swing feet, is shown by $h_{\mathrm{s}, v}(q) \in \mathbb{R}^{\bar{k}}$. We then have the constrained dynamics for $\mathcal{D}_{v}$ as:

$$
\left\{\begin{array}{l}
D(q) \ddot{q}+H(q, \dot{q})=B_{v} u+J_{v}^{T}(q) \lambda_{v} \\
J_{v}(q) \ddot{q}+\dot{J}_{v}(q, \dot{q}) \dot{q}=0
\end{array}\right.
$$

where $J_{v}(q):=\partial p_{v}(q) / \partial q$ represents the Jacobian matrix of the Cartesian position of the standing feet $p_{v}(q) \in \mathbb{R}^{3 k}$, with the corresponding constraint wrench $\lambda_{v} \in \mathbb{R}^{3 k}$. Note that the actuation matrix $B_{v}$ is domain dependent. This is because the double and triple support phases (Fig. 3) create closed-chain structures that induce redundancy in control and constraints. This is an underdetermined problem which often appears in multi-contact locomotion [32] that yields nonunique controllers for $u$. Therefore, we manually turn off the rear standing leg's hip pitch motor for double support phase and turn off the diagonal standing legs' hip pitch motors and the other standing leg's hip roll motors for triple supporting phase. This implementation in return yields underactauted dynamics for the full system. Now we can convert the constrained EOM (2) into a controlled ODE:

$$
\dot{x}=f_{v}(x)+g_{v}(x) u .
$$

To track a given set of time-based trajectories $\mathcal{B}_{v}(t)$, which will be detailed in a later section, we deployed an input output feedback linearization controller:

$$
u_{\text {io }}(x, t)=\mathcal{A}(x)\left(\mathcal{L}(x, t)-2 \varepsilon y(q, t)-\varepsilon^{2} \dot{y}(q, \dot{q}, t)\right) .
$$




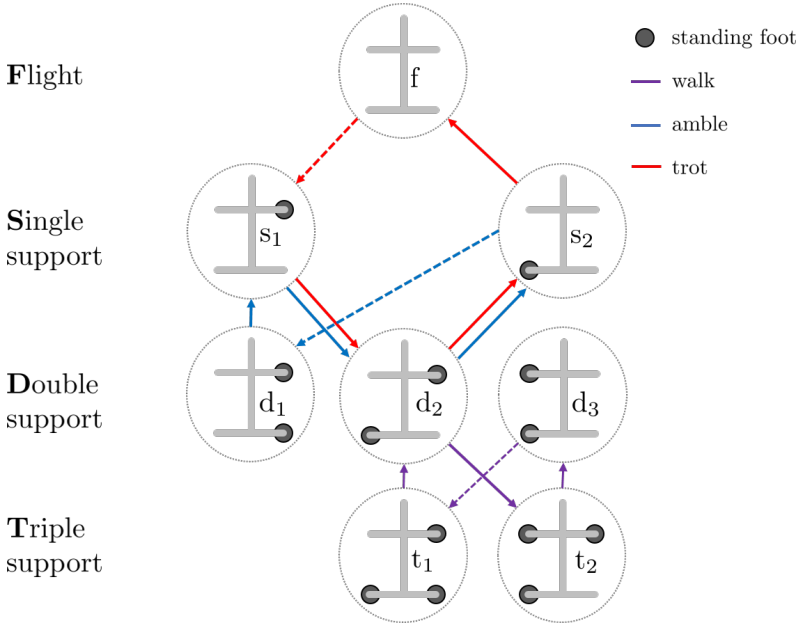

Fig. 3. The cyclic directed graph for the multi-domain hybrid dynamics of walking, ambling and trotting gaits. The dashed lines represent a relabeling map [8] that flips the left and right legs' contact attributes.

with the outputs $y(q, t)=y_{a}(q)-\mathcal{B}_{v}(t)$ and $\varepsilon>0$. In this formulation, we chose the actual outputs $y_{a}(q)$ as all of the actuated joints. The notations follow directly from Eq. (28) of [8]. The time-based controller has been justified for improved robustness over state-based methods both in theory [22] and experiments [23]. As a result, (4) forces the system to converge to a desired gait exponentially, that is, $y_{a}(q) \rightarrow \mathcal{B}_{v}(t)$. The output dynamics become

$$
\ddot{y}=-2 \varepsilon \dot{y}-\varepsilon^{2} y
$$

for which the origin $(y, \dot{y})=(0,0)$ is exponentially stable.

Prior to introducing the edges and the discrete dynamics, some assumptions are necessary to construct a feasible model both for computation and experiment:

- There is no ground slippage. This is partially guaranteed by enforcing a friction cone condition. However, slipping locomotion has been observed on quadrupedal animals for energy efficiency [13], [24].

- The ethology studies [25] have observed a pattern of $4 \times 2$-domain 1 locomotion on quadrupedal animals, and the authors have investigated this modeling method in [6]. But in this paper, we assume the stance leg transition domains -one leg strikes while another leg lifts are instantaneous and passive for walking and ambling. Hence $\mathbf{s}_{1}$ and $\mathbf{s}_{2}$ in Fig. 3 become edges and we can have a $2 \times 2$-domain behavior for walk and amble. But a $4 \times 2$-domain model is still used for trot.

\section{The discrete-time domain: impact and lift-off}

On the edge of $\mathcal{D}_{v}$ in (1), one of the conditions reaches its bound. Thus we have two switching mechanisms:

- Lift off: a standing foot of leg $l_{*}$ lifts off from the ground, meaning $N_{v}^{l_{*}}(x, u)=0$.

\footnotetext{
${ }^{1}$ The term $m \times 2$ denotes a gait with $m$-domain. See Fig. 3 for the directed graph of the gaits with $m$ domains. The second half of the motion is directly a left-right mirror (i.e., symmetry) of the gait. See Fig. 4 for the full motion with $m \times 2$ domains.
}

- Impact: a swing foot of leg $l_{*}$ impacts the ground, meaning $h_{s, v}^{l_{*}}(q)=0, \dot{h}_{s, v}^{l_{*}}(q, \dot{q})<0$.

For lift off, an identity map $x_{v+1}^{+}=x_{v}^{-}$is sufficient to represent the transition from current to the next domain, where $x_{v}^{-}$is the state at the end of the domain $\mathcal{D}_{v}$ and $x_{v+1}^{+}$ is the state at the beginning of $\mathcal{D}_{v+1}$. However, the impact shall cause a jump in the velocity terms. To describe this, we make use of a plastic impact model [15],

$$
\left[\begin{array}{cc}
D & -J_{v+1}^{T} \\
J_{v+1} & 0
\end{array}\right]\left[\begin{array}{c}
\dot{q}_{v+1}^{+} \\
\Lambda
\end{array}\right]=\left[\begin{array}{c}
D \dot{q}_{v}^{-} \\
0
\end{array}\right]
$$

by using the conservation of momentum while satisfying the next domain's holonomic constraints.

\section{HZD optimization}

An alternating sequence of the continuous dynamics (3) and the discrete dynamics (6) composites a hybrid control system. Since this nonlinear hybrid model has captured abundant details of the dynamics, its complexity challenges the controller design and motion planning. We hereby employ an optimization toolbox -FROST [17] that parses hybrid system control problems into a nonlinear programming (NLP) based on direct collocation. In this approach, to generate a feasible $N$-domain motion such as walking, ambling and trotting as shown in Fig. 3, the NLP is formulated as:

$$
\begin{aligned}
\min _{\alpha_{v}, x_{i}, \dot{x}_{i} u_{i}} & \sum_{i}\left\|u_{i}\right\|_{2}^{2} \quad i=1,2, \ldots \sum_{v=1}^{N} M_{v} \\
\text { s.t. } & \text { C1. closed-loop dynamics } \\
& \text { C2. hybrid \& periodic continuity } \\
& \text { C3. physical feasibility }
\end{aligned}
$$

where $M_{v}$ is the number of collocation points and $\alpha_{v}$ is the decision variable paramterizing the desired trajectory for domain $\mathcal{D}_{v}$. The cost function is to minimize the torque so that experiment implementation is achievable. The constraint C1 is from (3) and (5), and the constraint $\mathbf{C 2}$ is referring to the state continuity through each edge, which could be equipped with a discrete jump in states. $\mathbf{C} 3$ enforces conditions including $\left\|u_{i}\right\|_{\infty} \leq 50,\left(q_{i}, \dot{q}_{i}\right) \in \mathcal{X}$, foot clearance and the friction pyramid conditions, so that the optimal solution is experimental feasible.

Remark Due to many types of aleatoric uncertainty in the model, not every solution of the NLP can lead to experimental success. Furthermore, some constraint setup can be illposed that the NLP converges poorly. Some heuristics were used to mitigate the computation: 1. Efficiently producing closed-loop controllers is the core innovation of FROST, but it also boosts the complexity of the problem. Thus using an open-loop setup (optimization without a feedback controller) to warm start a closed-loop problem is effective. 2. Tuning constraints on the acceleration (force) terms is normally more effective than tuning positions. The appearance of a behavior aligns with human intuition better, but it can often be too restrictive for the optimization to converge. 


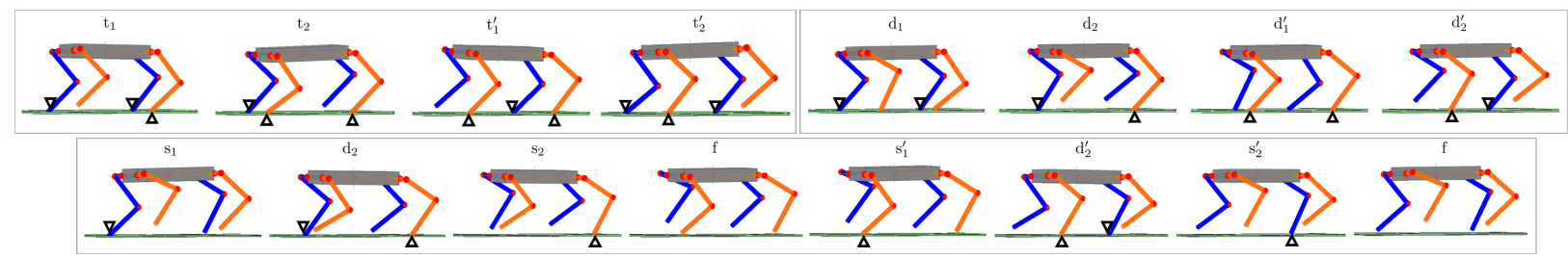

Fig. 4. On the top left is the snapshot for a $2 \times 2$-domain walking gait; on the top right is a $2 \times 2$-domain ambling gait; and on the bottom is a $4 \times 2$-domain trotting gait. The symbol $\square^{\prime}$ is a left-right mirror of the contact attributes for domain $\square$. The orange colored legs are the left side legs whose contact points are highlighted by triangles; the blue colored legs are the right side legs with contact points highlighted by inverted triangles.
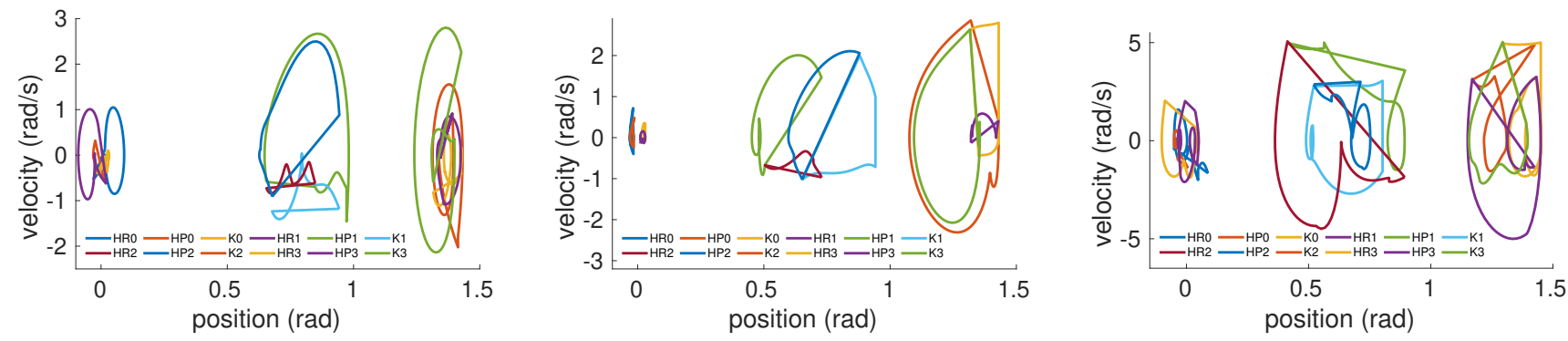

Fig. 5. The periodic trajectories designed by NLP 77: walk (the left), amble (the middle) and trot (the right).

\section{E. Optimal gaits}

Under the umbrella of the HZD framework, the sole difference among these behaviors in Fig. 3 is nothing but the ordered sequence of contact events, which can be predefined by specifying the stance foot clearance as 0 and swing foot clearance as nonzero values. Therefore, in the HZD optimization (7), by changing the foot clearance constraints in $\mathbf{C 3}$, multiple quadrupedal behaviors such as walk, amble and trot can be produced efficiently. We show the simulated behaviors in Fig. 4 and their phase portraits in Fig. 5 . With some initial guesses supplied, the computation time for the presented gaits are $262.13 \mathrm{~s}$ for walking, $42.69 \mathrm{~s}$ for ambling and $116.05 \mathrm{~s}$ for trotting on a Ubuntu 16.04 machine with Intel Core i7-6820 HQ CPU @ 2.7 GHz with 16 GB RAM.

\section{EXPONENTIAL STABILIZATION}

Quadrupedal dynamics are by nature contact-rich and high-dimensional. Therefore, including a Lyapunov-type stability criteria in the NLP is not computationally feasible. As a result, not every trajectory from the optimization is guaranteed to be a stable solution of the closed-loop system. In this section, we present an iterative algorithm that can postprocess the controller parameters from (7) to stabilize the resultant trajectories.

In the previous work [2], we have observed the stability of gaits in the HZD approach depends on the proper selection of the output functions to be regulated. Using the Poincaré sections analysis [14], our previous work [2], [3] has developed a recursive algorithm, based on BMIs, to systematically design output functions for which the gaits are exponentially stable for the corresponding closed-loop dynamics. The algorithm is offline and assumes a finitedimensional parameterization of the output functions to be determined. Then it translates the exponential stabilization problem into a recursive optimization problem that is set up based on LMIs and BMIs. The objective is to converge to a set of stabilizing parameters for which the given orbit (i.e., gait) is stable for the closed-loop system. Further, we assume that the outputs are parameterized by some controller parameters $\xi_{v}$ during the domain $v$, i.e., $y=y\left(q, t, \xi_{v}\right)$. For instance, $\xi_{v}$ can parameterize the controlled variables (see [2, Example 2]). Then, the evolution of the hybrid system on the Poincaré section can be described by

$$
x_{a}[k+1]=P_{a}\left(x_{a}[k], \xi\right), \quad k=0,1,2, \cdots,
$$

where $x_{a}:=\left(x^{\top}, t\right)^{\top}$ denotes the augmented states for the nonautonomous system and $P_{a}$ represents the parameters and augmented Poincaré return map [26]. Here, $\xi$ denotes the controller parameters over different domains of the directed cycle. The problem of exponential stabilization consists of designing the controller parameters such that the eigenvalues of the Jacobian matrix $A(\xi):=\frac{\partial P_{a}}{\partial x_{a}}\left(x_{a}^{\star}, \xi\right)$ lie inside the unit circle, were $x_{a}^{\star}$ represents the corresponding fixed point for the gait. The BMI algorithm consists of three steps: 1) sensitivity analysis, 2) BMI optimization, and 3) iteration. The sensitivity analysis generates a first-order approximation for the nonlinear Poincare map in terms of the controller parameters $\xi$ (see [2, Theorem 2]). This reduces the original exponential stabilization problem into BMIs and LMIs. More specifically, we translate the design problems of the original nonlinear system into BMIs for the first-order approximation of the Poincare map [3]. The local solutions of the BMI optimizer are then used to update the controller parameters for the next iteration [3], [5]. The algorithm continues until the spectral radius of the Jacobian linearization is less then a desired value. Sufficient conditions for the convergence of the algorithm to a set of stabilizing parameters in a finite number of iterations have been presented in our preliminary work [3]. By design, the BMI optimization problems can be solved effectively with available software packages such as PENBMI [2], [3], [4], [16]. 

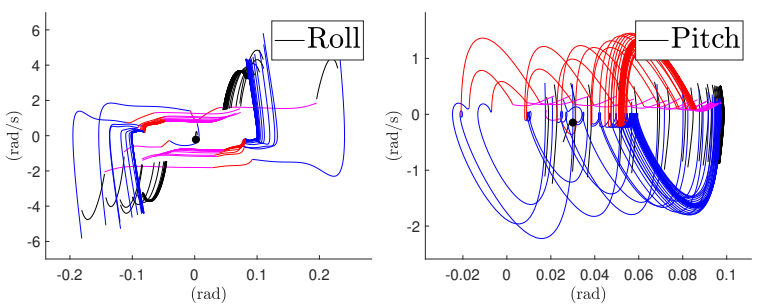

Fig. 6. Phase portraits during 50 consecutive steps of the $4 \times 2$ domains trot gait with the BMI-optimized virtual constraint controllers.

We now apply this algorithm to stabilize a 4-domain trotting gait. The dominant eigenvalues of the Jacobian matrix of the Poincaré map around the corresponding fixed point are $\{-1.1062,1.000,-0.4201+0.7278 i\}$ for a trotting gait generated by the NLP (7). To stabilize it, the iterative BMI algorithm modifies the output functions to be regulated. Here, the BMI algorithm looks for the controller parameters $\xi$ that parameterize controlled variables as $y_{a}\left(q, \xi_{v}\right)=H_{0}\left(\xi_{v}\right) q$, with $H_{0}\left(\xi_{v}\right)$ the output matrix that is parameterized by $\xi_{v}$. Starting from the nominal controlled variables, the BMI algorithm successfully converges to a stabilizing set of output parameters after 6 iterations for which the dominant eigenvalues of the Poincaré map become $\{0.8877,-0.1507 \pm 0.8661 i,-0.8830 \pm 0.0994 i\}$, which locally and exponentially stabilizes the trotting gait. From the phase portraits for the closed-loop hybrid system using the BMI-stabilized controller, shown in Fig. 6, convergence to the desired orbit is clear. We remark that each iteration of the BMI optimization takes approximately 30 minutes on a Windows machine with an Intel Xeon Gold 6130 CPU @ $2.10 \mathrm{GHz}$ and $64 \mathrm{~GB}$ RAM.

\section{EXPERIMENTS}

With these stabilized quadrupedal dynamic gaits in simulation and optimization, we conduct an experiment with the ambling gait on Vision 60 . The implemented controller is a PD approximation of the input-output linearizing controllers to track the time-based trajectories given by the optimization 77. That is, for a continuous domain $\mathcal{D}_{v}$, we have

$$
u\left(q_{a}, \dot{q}_{a}, t\right)=-K_{p}\left(q_{a}-\mathcal{B}_{v}(t)\right)-K_{d}\left(\dot{q}_{a}-\dot{\mathcal{B}}_{v}(t)\right)
$$

as the motor torque commands sent to each joint. The timebased PD implementation (9) has been shown to have exceptional robustness for bipedal locomotion [22]. In addition, the domain switching method is also time-based with the event function given by the optimized trajectories.

The result is that Vision 60 ambles stably with the desired speed of $0.3 \mathrm{~m} / \mathrm{s}$. See [1] for Vision 60 ambling in an outdoor tennis court and the snapshots are shown in Fig. 8. We logged 20 seconds of data and compared it with the simulated ambling controller in Fig. 7. Additionally, the average torque inputs (absolute value) are $7.73 \mathrm{~N} \cdot \mathrm{m}$ on the hip roll joints, $9.46 \mathrm{~N} \cdot \mathrm{m}$ on the hip pitch joints and $16.17 \mathrm{~N} \cdot \mathrm{m}$ on the knee joints. It is worth mentioning that the consistent drifting aside is expected, as there is no feedback information for the uneven terrain and that some
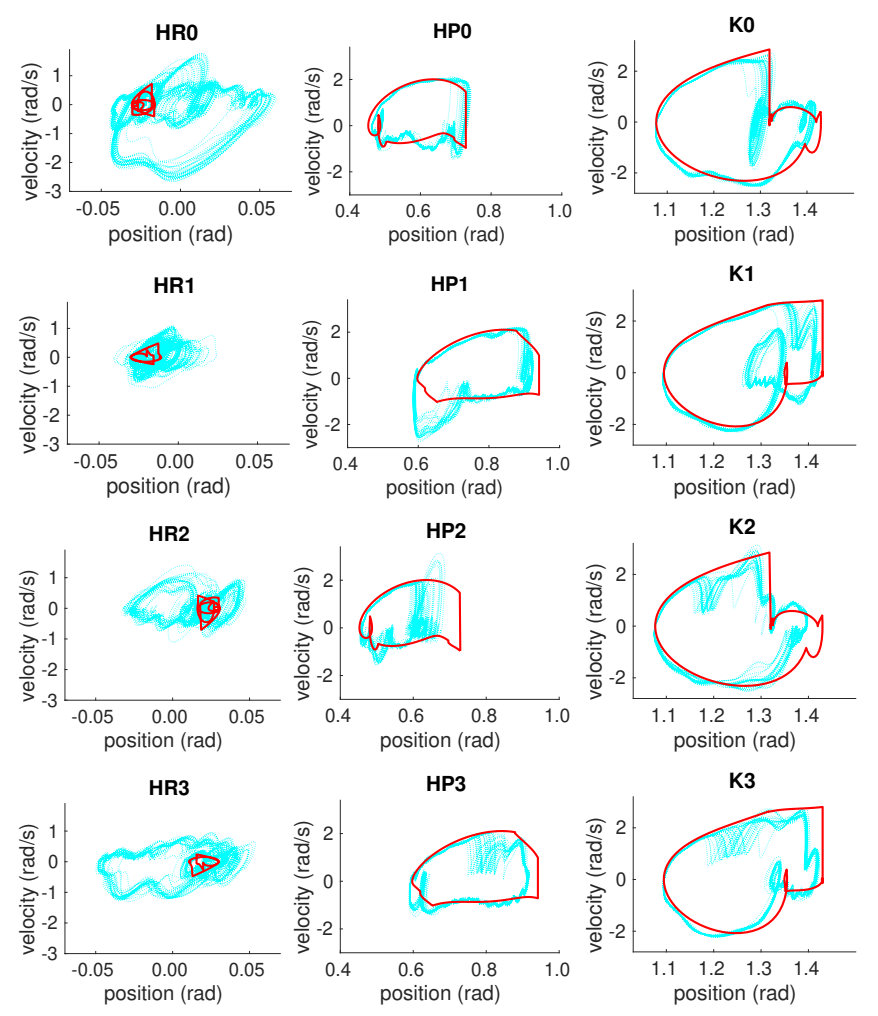

Fig. 7. The designed gaits (in red) from optimization/simulation vs. the experimental data (in cyan) in the form of phase portrait for amble. HR is short for hip roll, HP is for hip pitch and $\mathrm{k}$ is for knee.

manufacturing defects could cause asymmetric weight distribution. Some common solutions to avoid the drift is to use a joystick to manually offset the hip roll joints or Raibert-type regulators [29]. However, for the sake of showing a direct story from modeling and motion planning to experiments, we present the immediate implementation without any hidden layers of add-on controllers. The fact that the robot keeps ambling without falling supports the feasibility of the full model based HZD methods for quadrupedal locomotion.

\section{CONCLUSion}

In this paper, we adopted the HZD framework from bipedal to quadrupedal robots. This method systematically addresses 1) the construction of multi-domain hybrid models for dynamic locomotion, 2) full-order gait planning for agile behaviors - walk, amble and trot, and 3) HZD-based control synthesis to achieve dynamic stability and robustness. We also validated one of the behaviors via experiments on a quadrupedal robot with 36 state variables and 12 control inputs. The result is success ambling with Vision 60. We have shown the scalability and feasibility of the HZD approaches for the controls of quadrupedal locomotion, which initiates the first steps towards realizing more dynamical behaviors. The future work includes formally defining robustness for quadrupedal locomotion, and designing optimal controllers that can allocate force through actuators for the frequently appeared overactuated scenarios. 

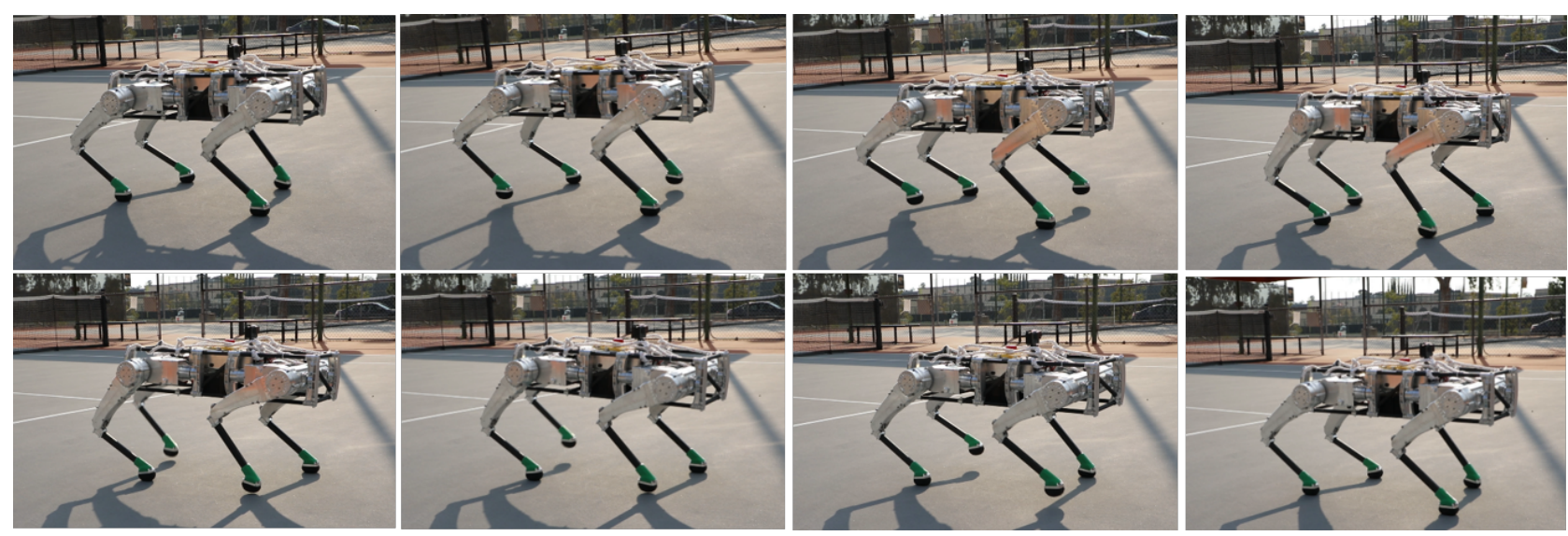

Fig. 8. Snapshots of the Vision 60 ambling in an outdoor environment, showing a full step of $2 \times 2$ domains of the amble gait.

\section{REFERENCES}

[1] Vision 60 Experiments, https://youtu.be/MQCPOKCop8Q

[2] K. Akbari Hamed, B. Buss, and J. Grizzle. Exponentially stabilizing continuous-time controllers for periodic orbits of hybrid systems: Application to bipedal locomotion with ground height variations. The International Journal of Robotics Research, 35(8):977-999, 2016.

[3] K. Akbari Hamed and R. D. Gregg. Decentralized feedback controllers for robust stabilization of periodic orbits of hybrid systems: Application to bipedal walking. Control Systems Technology, IEEE Transactions on, 25(4):1153-1167, July 2017.

[4] K. Akbari Hamed and R. D. Gregg. Decentralized event-based controllers for robust stabilization of hybrid periodic orbits: Application to underactuated $3 \mathrm{~d}$ bipedal walking. IEEE Transactions on Automatic Control, pages 1-16, July 2018.

[5] K. Akbari Hamed and J. Grizzle. Iterative robust stabilization algorithm for periodic orbits of hybrid dynamical systems: Application to bipedal running. In The IFAC Conference on Analysis and Design of Hybrid Systems, pages 161-168, Oct 2015.

[6] K. Akbari Hamed, W.-L. Ma, and A. D. Ames. Dynamically stable 3D quadrupedal walking with multi-domain hybrid system models and virtual constraint controllers. In American Control Conference, arXiv preprint arXiv:1810.06697, July 2019.

[7] A. Ames, K. Galloway, K. Sreenath, and J. Grizzle. Rapidly exponentially stabilizing control Lyapunov functions and hybrid zero dynamics. Automatic Control, IEEE Transactions on, April 2014.

[8] A. D. Ames. Human-inspired control of bipedal walking robots. IEEE Transactions on Automatic Control, 59(5):1115-1130, May 2014.

[9] C. Boussema, M. J. Powell, G. Bledt, A. J. Ijspeert, P. M. Wensing, and S. Kim. Online gait transitions and disturbance recovery for legged robots via the feasible impulse set. IEEE Robotics and Automation Letters, 4(2):1611-1618, April 2019.

[10] H. Dai and R. Tedrake. Optimizing robust limit cycles for legged locomotion on unknown terrain. In Decision and Control, IEEE 51st Annual Conference on, pages 1207-1213, Dec 2012.

[11] A. De and D. E. Koditschek. Vertical hopper compositions for preflexive and feedback-stabilized quadrupedal bounding, pacing, pronking, and trotting. The International Journal of Robotics Research, 37(7):743-778, 2018.

[12] S. A. J. v. der and J. M. Schumacher. Introduction to Hybrid Dynamical Systems. Springer-Verlag, Berlin, Heidelberg, 1999.

[13] B. Gamus and Y. Or. Analysis of dynamic bipedal robot walking with stick-slip transitions. In ICRA, pages 3348-3355, 2013.

[14] J. Grizzle, G. Abba, and F. Plestan. Asymptotically stable walking for biped robots: Analysis via systems with impulse effects. Automatic Control, IEEE Transactions on, 46(1):51-64, Jan 2001.

[15] J. W. Grizzle, C. Chevallereau, R. W. Sinnet, and A. D. Ames. Models, feedback control, and open problems of 3D bipedal robotic walking. Automatica, 50(8):1955 - 1988, 2014.

[16] D. Henrion, J. Lofberg, M. Kocvara, and M. Stingl. Solving polynomial static output feedback problems with PENBMI. In Decision and Control, and European Control Conference. 44th IEEE Conference on, pages 7581-7586, Dec 2005.
[17] A. Hereid, C. M. Hubicki, E. A. Cousineau, and A. D. Ames. Dynamic humanoid locomotion: A scalable formulation for HZD gait optimization. IEEE Transactions on Robotics, 2018.

[18] M. Hutter, C. Gehring, D. Jud, A. Lauber, C. D. Bellicoso, V. Tsounis, J. Hwangbo, K. Bodie, P. Fankhauser, M. Bloesch, R. Diethelm, S. Bachmann, A. Melzer, and M. Hoepflinger. Anymal - a highly mobile and dynamic quadrupedal robot. In 2016 IEEE/RSJ International Conference on Intelligent Robots and Systems (IROS), Oct 2016.

[19] A. Isidori. Nonlinear Control Systems. Springer; 3rd edition, 1995.

[20] A. M. Johnson, S. A. Burden, and D. E. Koditschek. A hybrid systems model for simple manipulation and self-manipulation systems. The International Journal of Robotics Research, 35(11):1354-1392, 2016.

[21] S. Kajita, K. Tani, and A. Kobayashi. Dynamic walk control of a biped robot along the potential energy conserving orbit. In IEEE International Workshop on Intelligent Robots and Systems, Towards a New Frontier of Applications, pages 789-794 vol.2, July 1990.

[22] S. Kolathaya, A. Hereid, and A. D. Ames. Time dependent control Lyapunov functions and hybrid zero dynamics for stable robotic locomotion. In 2016 American Control Conference (ACC), pages 3916-3921, July 2016.

[23] W.-L. Ma, S. Kolathaya, E. R. Ambrose, C. M. Hubicki, and A. D. Ames. Bipedal robotic running with durus-2d: Bridging the gap between theory and experiment. In Proceedings of the 20th International Conference on Hybrid Systems: Computation and Control, HSCC '17, pages 265-274, New York, NY, USA, 2017. ACM.

[24] W.-L. Ma, Y. Or, and A. D. Ames. Dynamic walking on slippery surfaces: Demonstrating stable bipedal gaits with planned ground slippage. In Robotics and Automation, IEEE International Conference on, May 20019.

[25] E. Muybridge. Animals in Motion. London:Chapman and Hall, LD.

[26] T. Parker and L. Chua. Practical Numerical Algorithms for Chaotic Systems. Springer, 1989.

[27] I. Poulakakis and J. Grizzle. The spring loaded inverted pendulum as the hybrid zero dynamics of an asymmetric hopper. Automatic Control, IEEE Transactions on, 54(8):1779-1793, Aug 2009.

[28] M. Raibert, K. Blankespoor, G. Nelson, and R. Playter. Bigdog, the rough-terrain quadruped robot. IFAC Proceedings Volumes, 41(2): 10822 - 10825, 2008. 17th IFAC World Congress.

[29] M. H. Raibert, H. B. Brown, and M. Chepponis. Experiments in balance with a $3 \mathrm{~d}$ one-legged hopping machine. The International Journal of Robotics Research, 3(2):75-92, 1984.

[30] K. Sreenath, H.-W. Park, I. Poulakakis, and J. W. Grizzle. Compliant hybrid zero dynamics controller for achieving stable, efficient and fast bipedal walking on MABEL. The International Journal of Robotics Research, 30(9):1170-1193, Aug. 2011.

[31] E. Westervelt, J. Grizzle, C. Chevallereau, J. Choi, and B. Morris Feedback Control of Dynamic Bipedal Robot Locomotion. Taylor \& Francis/CRC, 2007

[32] H.-H. Zhao, W.-L. Ma, M. B. Zeagler, and A. D. Ames. Humaninspired multi-contact locomotion with amber2. In Cyber-Physical Systems (ICCPS), 2014 ACM/IEEE International Conference on, pages 199-210, April 2014 